\title{
Eigenfunction expansions for generators of Dirichlet forms
}

\author{
Dedicated to the memory of Klaus Floret \\ By Anne Boutet de Monvel at Paris and Peter Stollmann at Chemnitz
}

\begin{abstract}
We present an eigenfunction expansion theorem for generators of strongly local, regular Dirichlet forms. Conditions are phrased in terms of the intrinsic metric. The result covers many cases of Hamiltonians which appear in Mathematical Physics and Geometry.
\end{abstract}

\section{Introduction}

The issue of expansion in generalized eigenfunctions is a classical one going back at least to Fourier. A standard reference is [1]. Apart from its structur theoretic value its development has been stimulated by applications to mathematical physics, as seen in [32], [29], [28] and the references there. A relatively recent impact is due to the study of wave propagation in random media [19], [24], [34], where eigenfunction expansions are an important input in the proof of localization. The use of this tool is settled by classical results in the Schrödinger operator case. But with the study of operators related with classical waves, [17], [33], a need for more general results on eigenfunction expansion became apparent. This is one of the sources from which we take our motivation. The other source is of quite different nature. It concerns the study of regular Dirichlet forms and their associated operators in terms of geometric properties of the underlying space that carries a natural metric which is induced by the Dirichlet form (once the latter is strongly local). The systematic exploration of this metric, often called the intrinsic metric, has led to quite a number of beautiful results which address questions of regularity of solutions, decay properties of heat kernels as well as spectral properties of the associated operator, [2], [4], [9], [38], [39], [40]. This associated operator is typically a kind of Laplacian and so the results of this genre have a wide range of applications and provide a unified treatment of quite a number of important cases including Laplacians on Riemannian manifolds, uniformly elliptic operators with weights, Hörmander type operators, subelliptic operators on euclidean space and Laplacians on graphs. Another advantage of the framework is that one can treat partial differential operators under minimal assumptions concerning the regularity of the coeffi- 
cients. Moreover, in many instances, the proofs found in the abstract setting are even easier than those known before.

In the present paper we contribute to this line of research, showing that generalized eigenfunction expansions can be obtained in the framework of strongly local, regular Dirichlet forms under quite general and natural assumptions.

The material is presented as follows: in Section 1 we outline the above mentioned framework. For the convenience of the reader, strong locality, the energy measure and the intrinsic metric are introduced along with the properties needed later. We also formulate a first result on generalized eigenfunction expansions in order to give an impression of the main results obtained here. Section 2 starts with an abstract Hilbert space theoretic result on generalized eigenfunction expansions from [29]. This is the starting point of our investigation and we will exploit the intrinsic geometry to provide the necessary input to use the result from [29]. Notably this includes exponential decay estimates for resolvents as well as a factorization result. Our main theorem is Theorem 2.5. In Section 3 we present a list of situations in which our result can be applied. In particular, the discussion of subelliptic operators in Subsection 3.2 covers as a very special case the random divergence form operators from [17], [33]. Moreover, we discuss the treatment of perturbations, even as singular as certain measure perturbations. This leads to a considerable extension of Theorem 2.5.

The second author wishes to express his sincere gratitude to the late Klaus Floret whose untimely death we deeply mourn. The present article would not have been written without his influence.

\section{The framework}

Many interesting operators in mathematical physics can be described in the framework of Dirichlet forms that we now briefly describe. A list of examples is given in 3 below. We follow here notation from [38] and [18], where details can be found. Consider a locally compact separable Hausdorff space $X$ endowed with a positive Radon measure $m$ with $\operatorname{supp} m=X$.

The basic object of our studies is a strongly local, regular Dirichlet form $\mathscr{E}$ with domain $\mathscr{D}$ in $L^{2}(X)$ and the selfadjoint operator $H$ associated with $\mathscr{E}$.

This means that $\mathscr{D} \subset L^{2}(X, m)$ is a dense subspace, $\mathscr{E}: \mathscr{D} \times \mathscr{D} \rightarrow[0, \infty)$ is bilinear and $\mathscr{D}$ is closed with respect to the energy norm $\|\cdot\|_{\mathscr{E}}$, given by

$$
\|u\|_{\mathscr{E}}^{2}=\mathscr{E}(u, u)+\|u\|_{L^{2}(X, m)}^{2}
$$

in which case one speaks of a closed form in $L^{2}(X, m)$. The unique operator $H$ associated with $\mathscr{E}$ is then characterized by

$$
D(H) \subset \mathscr{D} \quad \text { and } \quad \mathscr{E}(f, v)=(H f \mid v) \quad(f \in D(H), v \in \mathscr{D}) .
$$

Such a closed form is said to be a Dirichlet form if $\mathscr{D}$ is stable under certain operations; more precisely if for any $u \in \mathscr{D}$ also 


$$
u^{+}=u \vee 0=\max \{u, 0\}
$$

belongs to $\mathscr{D}$ and $\mathscr{E}\left(u^{+}, u^{+}\right) \leqq \mathscr{E}(u, u)$ and for any $v \in \mathscr{D}, \quad v \geqq 0$ the element $v \wedge 1=\min \{v, 1\} \in \mathscr{D}$ and $\mathscr{E}(v \wedge 1, v \wedge 1) \leqq \mathscr{E}(v, v)$.

A Dirichlet form is called regular if $\mathscr{D} \cap C_{\mathrm{c}}(X)$ is dense both in $\left(\mathscr{D},\|\cdot\|_{\mathscr{E}}\right)$ and $\left(C_{\mathrm{c}}(X),\|\cdot\|_{\infty}\right)$, where $C_{\mathrm{c}}(X)$ denotes the space of continuous functions with compact support.

$\mathscr{E}$ is strongly local if $\mathscr{E}(u, v)=0$ whenever $u$ is constant a.s. on the support of $v$.

The typical example one should keep in mind is the Laplacian $H=-\Delta$ on $L^{2}(\Omega)$, $\Omega \subset \mathbb{R}^{d}$ open, in which case $\mathscr{D}=W_{0}^{1,2}(\Omega)$ and $\mathscr{E}(u, v)=\int_{\Omega}(\nabla u \mid \nabla v) d x$. Now we turn to an important notion generalizing the measure $(\nabla u \mid \nabla v) d x$ appearing above.

In fact, every strongly local, regular Dirichlet form $\mathscr{E}$ can be represented in the form

$$
\mathscr{E}(u, v)=\int_{X} d \Gamma(u, v)
$$

where $\Gamma$ is a nonnegative symmetric mapping from $\mathscr{D} \times \mathscr{D}$ to the set of signed Radon measures on $X$. It is determined by

$$
\int_{X} \phi d \Gamma(u, u)=\mathscr{E}(u, \phi u)-\frac{1}{2} \mathscr{E}\left(u^{2}, \phi\right)
$$

and called energy measure; see also [3]. The energy measure satisfies the Leibniz rule,

$$
d \Gamma(u \cdot v, w)=u d \Gamma(v, w)+v d \Gamma(u, w)
$$

as well as the chain rule

$$
d \Gamma(\eta(u), w)=\eta^{\prime}(u) d \Gamma(u, w) .
$$

One can even insert functions from $\mathscr{D}_{\text {loc }}$ into $d \Gamma$, where $\mathscr{D}_{\text {loc }}=:\left\{u \in L_{\text {loc }}^{2}\right.$ such that $\phi u \in \mathscr{D}$ for all $\left.\phi \in \mathscr{D} \cap C_{\mathrm{c}}(X)\right\}$ to $d \Gamma$, as is readily seen from the defining equation (1.1) above. Using the energy measure one can define the intrinsic metric $\rho$ by

$$
\rho(x, y)=\sup \left\{|u(x)-u(y)|: u \in \mathscr{D}_{\mathrm{loc}} \cap C(X) \text { and } d \Gamma(u, u) \leqq d m\right\}
$$

where the latter conditions signifies that $\Gamma(u, u)$ is absolutely continuous with respect to $m$ and the Radon-Nikodym derivative is bounded by 1 on $X$. We assume throughout that $\rho$ induces the original topology on $X$ and that all balls with respect to $\rho$ are relatively compact in the original topology. This assumption is not very restrictive and well established for the applications we have in mind; see [38].

We single out two more requirements that are satisfied in typical finite dimensional situations. The first concerns $L^{p}$-mapping properties of the heat semigroup $e^{-t H}$ and reads

$$
e^{-t H}: L^{2} \rightarrow L^{\infty} \quad \text { for some } t>0 .
$$


The second condition signifies that the volume growth of balls $B_{R}(x)$ (with respect to the intrinsic metric) is slower than exponential.

$$
e^{-\alpha R} m\left(B_{R}\left(x_{0}\right)\right) \rightarrow 0 \quad \text { for every } \alpha>0
$$

Let us finally call $\phi \in \mathscr{D}_{\text {loc }} \backslash\{0\}$ a generalized eigenfunction for $H$ corresponding to $\lambda \in \mathbb{R}$, whenever

$$
\mathscr{E}(\phi, w)=\lambda(\phi \mid v) \quad \text { for all } v \in \mathscr{D} \cap C_{\mathrm{c}}(X)
$$

We now state the main result of the present article in a form considerably weaker than what we actually obtain in the next section:

Theorem 1.1. Let $\mathscr{E}, H$ be as above and assume (A1), (A2). Then, for spectrally almost every $\lambda \in \sigma(H)$ there exists a generalized eigenfunction $\phi$ of $H$ corresponding to $\lambda$ which is $L^{2}$-subexponentially bounded.

Here, $\phi$ is said to be $L^{2}$-subexponentially bounded if, for any $\alpha>0$,

$$
e^{-\alpha \rho\left(x_{0}, x\right)} \phi(x) \in L^{2}(X)
$$

for some $x_{0} \in X$. As we remarked above, we will be able to prove a stronger result. Two aspects are worthwhile mentioning here: we get a Fourier type expansion of arbitrary $L^{2}$-functions in terms of generalized eigenfunctions. As this involves the issue of ordered spectral representations we did not include it in the preceding theorem. The second improvement concerns the bound on generalized eigenfunctions. It can be sharpened according to the behaviour of the measure of the ball $B_{R}(x)$ for large $R$. In typical applications we have in mind, there is a bound of the form

$$
m\left(B_{R}(x)\right) \leqq c \cdot R^{d},
$$

in which case we get generalized eigenfunctions which obey a bound of the form

$$
\phi(x)\left(1+\rho\left(x_{0}, x\right)\right)^{-\frac{d+1}{2}-\varepsilon} \in L^{2}
$$

for every $\varepsilon>0$.

Let us now comment on the strategy of proof we choose. We use an abstract result [29] that yields a Fourier type expansion provided certain auxiliary operators satisfy Hilbert-Schmidt properties.

In our context, the respective Hilbert-Schmidt properties will follow by a factorization scheme using (A1), (A2). In the definition of the auxiliary operator the intrinsic metric will enter in a crucial way. It is used to define weight functions $w: X \rightarrow[1, \infty)$,

$$
w(y)=\omega\left(\rho\left(x_{0}, x\right)\right)
$$

where $\omega:[0, \infty) \rightarrow[1, \infty)$ is suitable chosen. In order to see that the Fourier type expansion consists of generalized eigenfunctions in the above sense we have to verify that $H$ admits a core of functions that decrease rapidly enough in terms of the weight function. 
This will involve a Combes-Thomas estimate or Davies' trick which amounts to showing that the resolvent of $H$ decays exponentially in the intrinsic metric.

\section{The expansion theorem}

For the reader's convenience, we first recall the abstract expansion theorem by Poerschke, Stolz and Weidmann [29] that serves as a starting point for our studies.

Given is a Hilbert space $(\mathscr{H},(\cdot \mid \cdot))$, a self-adjoint operator $H$ in $\mathscr{H}$ and an auxiliary closed operator $T \geqq 1$ in $\mathscr{H}$. With the help of the latter one defines the space

$$
\mathscr{H}_{+}:=\mathscr{H}_{+}(T):=D(T)
$$

that is a Hilbert space, endowed with the scalar product $(x \mid y)_{+}:=(T x \mid T y)$. If we dualize $\mathscr{H}_{+}$over the scalar product $(\cdot \mid \cdot)$ of $\mathscr{H}$ we get $\mathscr{H}_{-}:=\mathscr{H}_{-}(T)$, the completion of $\mathscr{H}$ with respect to the scalar product $(x \mid y)_{-}:=\left(T^{-1} x \mid T^{-1} y\right)$. Note that

$$
\mathscr{H}_{+} \subset \mathscr{H} \subset \mathscr{H}_{-} .
$$

A further important ingredient is an ordered spectral representation of $H$. This is a special form of representing a selfadjoint operator as a multiplication operator. It is particularly useful whenever one is interested in spectral multiplicities. We do not want to enter this latter subject here and refer the interested reader to [29] and the literature quoted there. Before stating the properties of an ordered spectral representation let us recall that a measure $\mu$ on $\mathbb{R}$ is called a spectral measure for $H$ if $\mu(A)=0$ if and only if $E_{H}(A)=0$, where $E_{H}$ denotes the spectral resolution of $H$. Given such a $\mu, N \in \mathbb{N} \cup\{\infty\}$ and a sequence of measurable subsets $M_{j} \subset \mathbb{R}$ such that $M_{j} \supset M_{j+1}$, a unitary mapping

$$
U=\left(U_{j}\right): \mathscr{H} \rightarrow \bigoplus_{j=1}^{N} L^{2}\left(M_{j}, d \mu\right)
$$

is said to be an ordered spectral representation of $H$ if

$$
U \varphi(H)=M_{\varphi} U,
$$

for every measurable function $\varphi$ on $\mathbb{R}$.

This means that $U$ "maps" $H$ to the operator of multiplication by the identity. Clearly, $\bar{M}_{1}=\sigma(H)$. Every selfadjoint operator admits an ordered spectral representation, see [29] and the references there.

Theorem 2.1. Let $H, T, \mathscr{H}_{+}, \mathscr{H}_{-}$be as above. Let $\mu$ be a spectral measure for $H$ and $U$ an ordered spectral representation. Assume that there is $\gamma \in C_{b}(\mathbb{R}, \mathbb{C})$ with $|\gamma|>0$ on $\sigma(H)$ such that $\gamma(H) T^{-1}$ is a Hilbert-Schmidt operator. Then there are measurable functions $\varphi_{j}: M_{j} \rightarrow \mathscr{H}_{-}$for $j=1, \ldots, N$ such that:

(1) $U_{j} f(\lambda)=\left(f \mid \varphi_{j}(\lambda)\right)$ for $f \in \mathscr{H}_{+}$and $\mu$-a.e. $\lambda \in M_{j}$. 
(2) For every $g=\left(g_{j}\right) \in \bigoplus_{j} L^{2}\left(M_{j}, d \mu\right)$ we have

$$
U g=\lim _{\substack{n \rightarrow N \\ E \rightarrow \infty}} \sum_{j=1}^{n} \int_{M_{j} \cap[-E, E]} g_{j}(\lambda) \varphi_{j}(\lambda) d \mu(\lambda)
$$

and, therefore, for every $f \in \mathscr{H}$

$$
f=\lim _{\substack{n \rightarrow N \\ E \rightarrow \infty}} \sum_{j=1}^{n} \int_{M_{j} \cap[-E, E]}\left(U_{j} f\right)(\lambda) d \mu(\lambda) .
$$

(3) If $f \in\left\{g \in D(H) \cap \mathscr{H}_{+}: H g \in \mathscr{H}_{+}\right\}$we get that

$$
\left(H f \mid \varphi_{j}(\lambda)\right)=\lambda\left(f \mid \varphi_{j}(\lambda)\right) \quad \text { for } \mu \text {-a.e. } \lambda \in M_{j} \text {. }
$$

Let us add a few comments: note first that the integrals

$$
\int_{M_{j} \cap[-E, E]} g_{j}(\lambda) \varphi_{j}(\lambda) d \lambda
$$

appearing in equations $(2.1)$ and (2.2) represent elements of $\mathscr{H}$, although $\varphi_{j}(\lambda) \in \mathscr{H}_{-}$only. This is reminiscent of the Fourier decomposition. In view of that analogy we speak of a Fourier type expansion if $\left(\varphi_{j}\right)$ satisfy (1) and (2) from the above theorem. It is clear that the term generalized eigenfunction expansion is justified whenever there are sufficiently many functions that obey (2.3), i.e., when

$$
\left\{g \in D(H) \cap \mathscr{H}_{+}: H g \in \mathscr{H}_{+}\right\}
$$

is an operator core for $H$.

As we already indicated in the preceding section, we shall use the above theorem for $\mathscr{H}=L^{2}(X, m)$ and $H$ the operator associated with a strongly local regular Dirichlet form.

We will choose $T=M_{w}$, multiplication with a weight function $w: X \rightarrow[1, \infty)$ that comes with the intrinsic metric $\rho$ and depends on the volume growth of balls as well. Moreover, $\gamma$ from the theorem will just be $e^{-s}$.

The Hilbert-Schmidt property for $\gamma(H) T^{-1}$ will follow easily from a factorization principle that has its roots in Grothendieck's work, see [13], [21]. It has been introduced for questions of the type considered here in [35], see also [14] for a simple proof in a special case. The second author learned a lot about these techniques from Klaus Floret, to whose memory the present paper is dedicated.

Factorization principle. Given two bounded operators $A \in \mathscr{B}\left(L^{2}, L^{\infty}\right)$ and $B \in \mathscr{B}\left(L^{\infty}, L^{2}\right)$, the product $B A$ is a Hilbert-Schmidt operator.

In our case $A=e^{-t H}, B=T^{-1}$ and, additionally, $A$ maps nonnegative functions to nonnegative functions. Therefore, from [14] it follows that $B A$ and consequently 
$A B=\gamma(H) T^{-1}$ are Hilbert-Schmidt, once we have chosen $w$ in such a way that $w^{-1} \in L^{2}$. Let us be precise on that first step:

Proposition 2.2. (1) Assume (A1). Then there exists a Fourier type expansion $\left(\varphi_{j}\right)$ of $H$. If $w: X \rightarrow[1, \infty)$ satisfies $w^{-1} \in L^{2}$, then $\left(\varphi_{j}\right)_{j}$ can be chosen such that

$$
w^{-1} \varphi_{j}(\lambda) \in L^{2} \quad \text { for } \mu \text {-a.e. } \lambda .
$$

(2) If, furthermore, (A2) is valid, we find an $L^{2}$-subexponentially bounded weight function $w \geqq 1$ such that $w^{-1} \in L^{2}$.

Proof. (1) By (A1) we have $e^{-t H}: L^{2} \rightarrow L^{\infty}$ for some $t>0$. Fix such a $t$ and let $\gamma(s):=e^{-t s} \wedge 1$ which satisfies the assumptions of Theorem 2.1. Moreover, $\gamma(H)=e^{-t H}$ since $\gamma(s)=e^{-t s}$ on $\sigma(H)$. Let $\omega:[0, \infty) \rightarrow[1, \infty)$ be a continuous, monotone increasing function such that

$$
\sum_{n} \omega(n)^{-2} m\left(B_{n+1}\left(x_{0}\right)\right)<\infty,
$$

where $x_{0} \in X$ is fixed. Then, obviously,

$$
w(x):=\omega\left(\rho\left(x_{0}, x\right)\right)+1
$$

is a weight function on $X$ with $w^{-1} \in L^{2}(X)$. Consequently, $T=M_{w}$ gives rise to

$$
\gamma(H) T^{-1}=\left(T^{-1} \gamma(H)\right)^{*}=\left(M_{w^{-1}} e^{-t H}\right)^{*} .
$$

This is a Hilbert-Schmidt operator by [14] as it factors through $L^{\infty}(X)$.

Theorem 2.1 yields a Fourier type expansion $\left(\varphi_{j}\right)$ with $\varphi_{j}(\lambda)=\left\{g: w^{-1} g \in L^{2}\right\}$ for $\mu$-a.e. $\lambda$. This finishes the proof of part (1).

Part (2) is easy, since the subexponential growth of $m\left(B_{R}\left(x_{0}\right)\right)$ allows to choose $w$ accordingly.

It is apparent that the volume growth of balls, (A2) does not enter the question of existence of a Fourier type expansion; it is needed only to control the growth of the $\varphi_{j}(\lambda)$.

As we shall see now, it can also be used to deriving that the condition of Theorem 2.1 (3) holds for an operator core of $H$. This ensures that the $\varphi_{j}(\lambda)$ obtained above are generalized eigenfunctions.

As a step in this direction let us now state that the resolvent of $H$ exhibits exponential decay off the diagonal. Estimates of this genre are quite useful in Mathematical Physics and often named Combes-Thomas estimates [5] in the respective literature. In different communities they are also known as Davies' trick in view of the method introduced in [9].

Proposition 2.3. Let $H$ be as above. Then there exist $C, \alpha>0$ such that for all $A, B \subset X, \lambda \geqq 1$ we have 


$$
\left\|\chi_{A}(H+\lambda)^{-1} \chi_{B}\right\| \leqq C \cdot e^{-\alpha \cdot \rho(A, B)}
$$

where $\rho(A, B)=\inf \{\rho(x, y): x \in A, y \in B\}$ and $\|\cdot\|$ denotes the operator norm.

Proof. One could use Davies' trick taking into account the properties of the energy measure. Such a simple approach yields, essentially, $\alpha=\lambda$ and $C=\lambda^{-1}$. Presumably this can be sharpened to $\alpha=\sqrt{\lambda}$ but we do not enter this issue here. However, we can also simply refer to [4], Corollary 3.

We get the following easy consequence:

Proposition 2.4. Assume (A2); let $w$ be an $L^{2}$-subexponentially bounded weight function. Then $\left\{g \in D(H): w f \in L^{2}, w H f \in L^{2}\right\}$ is an operator core for $H$.

Proof. Consider $D_{0}:=(H+1)^{-1} C_{\mathrm{c}}(X)$. Since $C_{\mathrm{c}}(X)$ is dense in $L^{2}, D_{0}$ is an operator core for $H$. By the Combes-Thomas estimate, Proposition 2.3, each $f \in D_{0}$ is exponentially decreasing. Hence $w f \in L^{2}$ by the growth condition on $w$ and

$$
w H f=w(H+1) f-w f=w(H+1)(H+1)^{-1} \varphi-w f=w \varphi-w f \in L^{2},
$$

since $f=(H+1)^{-1} \varphi$ with suitable $\varphi \in C_{\mathrm{c}}(X)$.

We can now state our main result.

Theorem 2.5. Assume (A1), (A2). Let $\mu$ be a spectral measure for $H$. Let $w: X \rightarrow[1, \infty)$ be a weight function such that $w^{-1} \in L^{2}(X)$ and $w$ is $L^{2}$-subexponentially bounded. Then there exists a Fourier type expansion $\left(\varphi_{j}\right)$ for $H$ such that for $\mu$-a.e. $\lambda \in \sigma(H)$ the function $\varphi_{j}(\lambda)$ is a generalized eigenfunction of $H$ for $\lambda$ with $w^{-1} \varphi_{j}(\lambda) \in L^{2}$.

Proof. By Proposition 2.2 we get a Fourier type expansion $\left(\varphi_{j}\right)$ such that $w^{-1} \varphi_{j}(\lambda) \in L^{2}$ for $\mu$-a.e. $\lambda \in \sigma(H)$. By Theorem 2.1(3) we know that

$$
\left(\varphi_{j}(\lambda) \mid H f\right)=\lambda\left(\varphi_{j}(\lambda) \mid f\right) \quad \mu \text {-a.e. }
$$

for $f \in\left\{g \in D(H): w, w H g \in L^{2}\right\}=: D_{w}$. This latter space is a core for $H$ according to Proposition 2.4 so (2.4) extends to

$$
\mathscr{E}\left(\varphi_{j}(\lambda), v\right)=\lambda\left(\varphi_{j}(\lambda) \mid v\right)
$$

for all $v \in \mathscr{D} \cap C_{c}(X)$. In fact, let us abbreviate $\phi:=\varphi_{j}(\lambda)$. We have to check that $\phi \in \mathscr{D}_{\text {loc }}$, i.e., that $\chi \phi \in \mathscr{D}$ for any cutoff $\chi \in \mathscr{D} \cap C_{\mathrm{c}}(X)$. First note that $(H+E)^{-1} \chi H^{\frac{1}{2}}$ extends to a bounded operator on $L^{2}(X)$. Pick an arbitrary $g \in D(H)$ and use the fact that $D_{w}$ is a core to get a sequence $\left(f_{n}\right) \in D_{w}$ that converges to $(H+E)^{-1} \chi H g$.

It follows that

$$
\left(\phi \mid(H+E) f_{n}\right)=(\lambda+E)\left(\phi \mid f_{n}\right)
$$

and for $n \rightarrow \infty$ we get 


$$
(\phi \mid \chi H g)=(\lambda+E)\left(\phi \mid(H+E)^{-1} \chi H g\right) .
$$

Therefore,

$$
|(\chi \phi \mid H g)|=|(\phi \mid \chi H g)| \leqq c_{\chi}\left\|H^{\frac{1}{2}} g\right\|
$$

In particular, $\chi \phi \in \mathscr{D}$.

Now every $u \in \mathscr{D} \cap C_{\mathrm{c}}(X)$ can be approximated by a sequence $\left(g_{n}\right)$ from $D_{w}$ in $\mathscr{E}$-norm. This gives

$$
\mathscr{E}(\phi, u)=\lim _{n} \mathscr{E}\left(\phi, g_{n}\right)=\lim _{n} \lambda\left(\phi \mid g_{n}\right)=\lambda(\phi \mid u)
$$

Note that the choice of $w$ has two aspects: in order to get $w^{-1} \in L^{2}$ we might want to take $w$ very large near infinity. However, the resulting condition on the bound of $\varphi_{j}(\lambda)$ viz. $w^{-1} \varphi_{j}(\lambda) \in L^{2}$ will be weak then. Thus we should choose $w$ as small as possible near infinity subject to the condition $w^{-1} \in L^{2}$, of course.

If one has a better control on the volume growth of intrinsic balls, e.g., an estimate like

$$
m\left(B_{R}\left(x_{0}\right)\right) \leqq C \cdot R^{d}
$$

one gets better estimates for $\varphi_{j}(\lambda)$, e.g.,

$$
\left(1+\rho\left(x_{0}, x\right)\right)^{-\frac{d+1}{2}-\varepsilon} \varphi_{j}(\lambda) \in L^{2},
$$

which is the bound in many $d$-dimensional situations.

\section{Applications}

In this section we record a list of relevant examples that are covered by our result above. In fact, this work developed from an attempt to give a short and elegant proof of the existence of an eigenfunction expansion for random divergence form operators, see [17], [23], [24], [33]. This special case will be included in Subsection 3.2.

In the last subsection we will also describe how to pass from $H$ to $H+V$ where $V$ is a potential or, more generally, a measure perturbation.

3.1. Laplace-Beltrami operators on Riemannian manifolds. We refer to [9] for the necessary background and the results needed.

If $M$ is an $n$-dimensional complete Riemannian manifold, the Laplace-Beltrami operator $H=-\Delta$ is associated with a strongly local, regular Dirichlet form.

The heat semigroup $e^{-t H}$ satisfies Gaussian estimates in this case and is consequently ultracontractive, i.e., 


$$
e^{-t H}: L^{2} \rightarrow L^{\infty}
$$

for every $t>0$ so that (A1) is satisfied.

Concerning condition (A2) note that the intrinsic metric coincides with the Riemannian metric and that the volume growth of balls is governed by

$$
m\left(B_{R}(x)\right) \leqq C \cdot R^{n}
$$

provided the Ricci curvature is nonnegative. Therefore, by Theorem 2.5 and the remark following it we get a generalized eigenfunction expansion with at most polynomially growing eigenfunctions. More generally, if

$$
\left.\lim _{R \rightarrow \infty} \inf \operatorname{Ricci|}\right|_{B_{R}(x)} \geqq 0
$$

then (A2) is satisfied.

3.2. Subelliptic operators and operators of Hörmander type. For the necessary background on subelliptic operators see [8], [15], [16], [22], [26], [30]. Here we restrict our attention to the case $\mathbb{R}^{d}$. Consider a locally integrable function $\mathbf{a}: \mathbb{R}^{d} \rightarrow \mathbb{C}^{d \times d}$ such that $\mathbf{a}(x) \geqq 0$. We moreover assume that $\mathbf{a}$ is bounded. If

$$
\int(\mathbf{a}(x) \nabla u(x) \mid \nabla u(x)) d x+\|u\|^{2} \geqq C \cdot\|u\|_{s, 2}^{2}
$$

for some $s>0, c>0$ and all $u \in C_{\mathrm{c}}^{\infty},\|\cdot\|_{s, 2}$ denoting the norm in the Sobolev space of order $s$ over $L^{2}$, we call $H=\nabla \cdot \mathbf{a} \cdot \nabla$ subelliptic; here $H$ is the operator associated with the form

$$
\mathscr{E}(u, v)=\int(\mathbf{a}(x) \nabla u(x) \mid \nabla v(x)) d x .
$$

First note that for strictly positive matrix functions, i.e., when

$$
(\mathbf{a}(x) \xi \mid \xi) \geqq \eta\|\xi\|^{2}
$$

for some fixed positive $\eta$, the subellipticity condition is satisfied with $s=1$.

Using Nash's inequality, see [9], we get that every subelliptic operator $H=-\nabla \cdot \mathbf{a} \cdot \nabla$ satisfies (A1). Moreover, the intrinsic metric defined in this way has been studied extensively; cf. [15], [16], [22], [26]. (A2) is trivially satisfied since by assumption a is bounded and thus intrinsic balls of radius $R$ are contained in Euclidean balls of radius $C \cdot R$.

Of course, all the ingredients needed in the strictly elliptic case are very easy so we get the results needed for random operators, almost for free. Compare this with analogous results in the recent [23], [24] for somewhat more complicated operators. The generalization to operators on Lie groups can also be considered in the framework of the present paper. The relevant notions for such a generalization as well as a thorough analysis of subelliptic operators in the Lie group setting can be found in [30].

3.3. Laplacians on graphs. We follow here the notation from [25]. Let $X$ be some countable set of vertices and $E \subset X \times X$ the set of edges of a discrete graph that is assumed to be symmetric. Suppose we are given a weight function $b: E \rightarrow(0, \infty)$ such that 
sup $b<\infty$ and that we have a uniform bound $M$ on the number of edges emerging from a vertex, i.e.,

$$
M=\sup _{x \in X} \#\{(x, y): y \in X \text { and }(x, y) \in E\}<\infty .
$$

We can take as $H$ the Laplacian on $\ell^{2}(X)$ defined by

$$
H f(x)=\sum_{y \in X,(x, y) \in E} b((x, y))(f(y)-f(x)) .
$$

Assuming that $b(x, y)=b(y, x)$ for all $(x, y) \in E$ this operator is seen to come with a regular Dirichlet form. Moreover, since $\ell^{2} \subset \ell^{\infty}(X)$ assumption (A1) is satisfied. Although the form of $H$ is not strongly local, there is an appropriate intrinsic metric, see [11], [27], [25]. If (A2) is satisfied, we consequently get the analogue of Theorem 2.5.

For more recent and more precise estimates, see [6], [7], [20] and the references cited there.

3.4. Singular perturbations. Let us now turn back to the abstract case of a strongly local, regular Dirichlet form $\mathscr{E}$ on $X$. We want to discuss the question whether $H$ can be replaced by $H+V$ where $V$ is a perturbation with some mild regularity assumption. The answer will be yes and the necessary analysis is available from [37]. We refer to the literature quoted there and start to introduce the necessary notions.

We treat here "potentials" $V$ that may well be measures and include Dirichlet boundary conditions.

Consider the positive cone

$\{v \mid v$ a positive measure on $X$ such that capa $(B)=0$ implies $v(B)=0\}:=\mathscr{M}_{0}$

where cap is the capacity; see [18].

For every $v_{+} \in \mathscr{M}_{0}$ (in contrast to [37] we denote measures by $v$ rather than $\mu$ in order to distinguish them from the spectral measure $\mu$ from Section 2) we get an operator $H+v_{+}$ by form methods. This operator is defined on $L^{2}(Y)$ where $Y \subset X$ might be smaller than $X$ but the resolvent and the semigroup of $H$ act on $L^{2}(X)$ (they are 0 on $L^{2}(X \backslash Y)$ ). Moreover we can add a negative $v_{-}$from the generalized Kato class $\hat{\mathscr{S}}_{K}$ provided the Kato constant $c_{K}\left(v_{-}\right)<1$; see [37] for details. In that case $H+v_{+}-v_{-}$is bounded below and, due to the results from [37] (A1) is satisfied for $v_{+}-v_{-}$whenever it is satisfied for $H$. To construct an eigenfunction expansion we work with the intrinsic metric of unperturbed operator $H$ and get:

Corollary 3.1. Let $H, v_{+}, v_{-}$be as above and assume that (A1), (A2) are satisfied. Then the assertion of Theorem 2.5 remains valid for $H$ replaced by $H+v_{+}-v_{-}$.

Proof. Due to [37], Theorem 5.1, property (A1) is valid for $H+v, v=v_{+}-v_{-}$ under the assumptions made in the corollary. Therefore, we get a Fourier type expansion $\left(\varphi_{j}\right)$ for $H+v$ by Proposition 2.2. 
It remains to produce a weight function $w \geqq 1$ such that $w^{-1} \in L^{2}$. To this end we can use the one defined in the proof of Proposition 2.2, where $\rho$ is the intrinsic metric of the unperturbed operator $H$.

The form of $H+v$ is small with respect to the form of $H$, see e.g., [37], Theorem 3.1, so that Propositions 2.3 and 2.4 extend to the perturbed case. Proceeding like in the proof of Theorem 2.5 we get the assertion.

Remarks 3.2. (1) For singular perturbations it may happen that there are no nontrivial continuous functions in the form domain of $H+v$, see [36]. One should therefore point out that the identity

$$
\mathscr{E}\left(\varphi_{j}(\lambda), v\right)=\lambda\left(\varphi_{j}(\lambda) \mid v\right)
$$

extends to all bounded $v$ in the form domain that have compact support.

(2) Starting from a strongly regular Dirichlet form with operator $H$ and a measure $v_{+} \in \mathscr{M}_{0}$ it is clear that $H+v_{+}$corresponds to a Dirichlet form as well. Moreover, the semigroup has even better contraction properties than the unperturbed, as it is dominated pointwise by the latter. However, one looses the regularity of the form as already mentioned above so that the intrinsic metric for the perturbed form cannot be defined as above. Nevertheless, one might be able to find a well-behaved weight function for the perturbed rather than for the unperturbed operator. The referee raised the interesting question whether this is the case for $H$ the negative Laplace-Beltrami operator on hyperbolic space perturbed with a quadratic potential. For a decent treatment one should extend the present framework, a task we will not pursue here.

(3) In connection with the last remark note that the operator $H+v, v=v_{+}-v_{-}$ considered in the corollary does not correspond to a Dirichlet form, due to the negative perturbation. It is therefore reasonable to use the intrinsic metric of the unperturbed operator.

We should stress that this corollary now applies to all the classes of operators discussed in Subsections 3.1 to 3.3 above. This means that we get a fairly complete picture that includes most of the operators of Schrödinger type met in Mathematical Physics or Geometry all treated within the same framework.

Acknowledgement. The second author gratefully acknowledges invitations to the University Paris 7 Denis Diderot where part of this work was done and support by the DFG in the priority program "Interacting stochastic systems of high complexity". Moreover, we would like to thank the referee for helpful remarks.

\section{References}

[1] J. M. Berezanskii, Expansions in Eigenfunctions of Selfadjoint Operators, Trans. Math. Monogr. 17, Amer. Math. Soc., Providence 1968.

[2] M. Biroli and U. Mosco, A Saint-Venant principle for Dirichlet forms on discontinuous media, Ann. Mat. Pura Appl. (IV) 169 (1995), 125-181.

[3] N. Bouleau and F. Hirsch, Dirichlet forms and analysis on Wiener space, de Gruyter Stud. Math. 14, Berlin 1991. 
[4] F. Cipriani and G. Grillo, $L^{p}$-exponential decay for solutions to functional equations in local Dirichlet spaces, J. reine angew. Math. 496 (1998), 163-179.

[5] J.-M. Combes and L. Thomas, Asymptotic behaviour of Eigenfunctions for Multi-Particle Schrödinger Operators, Commun. Math. Phys. 34 (1973), 251-263.

[6] T. Coulhon and A. Grygoryan, Random walks on graphs with regular volume growth, Geom. Funct. Anal. 8 (1998), 656-701.

[7] T. Coulhon and A. Grygoryan, Pointwise estimates for transition probabilities of random walks on infinite graphs, Fractals in Graz, W. Woess, P. Grabner, eds., Birkhäuser, to appear.

[8] T. Coulhon, L. Saloff-Coste and N. Th. Varopoulos, Analysis and Geometry on Groups, Cambridge Tracts Math. 100, Cambridge University Press, Cambridge 1992.

[9] E. B. Davies, Explicit constants for Gaussian upper bounds on heats kernels, Amer. J. Math. 109 (1987), 319-334.

[10] E. B. Davies, Spectral Theory, Cambridge University Press, Cambridge 1989.

[11] E. B. Davies, Large deviations for heat kernels on graphs, J. London Math. Soc. (2) 47 (1993), 65-72.

[12] E. B. Davies, Analysis on graphs and noncommutative geometry, J. Funct. Anal. 111 (1993), 398-430.

[13] A. Defant and K. Floret, Tensor norms and operator ideals, North Holland Math. Stud. 176, Amsterdam 1993.

[14] M. Demuth, P. Stollmann, G. Stolz and J. Van Casteren, Trace norm estimates for products of integral operators, and diffusion semigroups, Integral Equ. Oper. Th. 23, n² (1995), 145-153.

[15] C. Fefferman and D. H. Phong, Subelliptic eigenvalue problems, in: Conference on Harmonic Analysis in honor of Antoni Zygmund, vol. 2, Wadsworth (1983), 590-606.

[16] C. Fefferman and A. Sanchez-Callé, Fundamental Solutions for Second Order Subelliptic Operators, Ann. Math. 124 (1986), 247-272.

[17] A. Figotin and A. Klein, Localization of classical waves I: Acoustic waves, Commun. Math. Phys. 180 (1996), 439-482.

[18] M. Fukushima, Dirichlet forms and Markov processes, North-Holland Mathematical Library 23, NorthHolland Publishing Co., Amsterdam-New York-Kodansha, Ltd.-Tokyo 1980.

[19] F. Germinet and A. Klein, Bootstrap Multiscale Analysis and Localization in Random Media, Commun. Math. Phys. 222 (2001), 415-448.

[20] A. Grigor'yan and A. Telcs, Sub-Gaussian estimates of heat kernels on infinite graphs, Duke Math. J. 109 (2001) no. 3, 452-510.

[21] A. Grothendieck, Résumé de la théorie métrique des produits tensoriels topologiques, Bol. Soc. Mat. Sao Paulo 8 (1956), 1-79.

[22] D. Jerison and A. Sanchez-Callé, Subelliptic, second order differential operators, Complex analysis, III (College Park, Md., 1985-86), Springer Lect. Notes Math. 1277 (1987), 46-77.

[23] A. Klein and A. Koines, A general framework for localization of classical waves. I. Inhomogeneous media and defect eigenmodes, Math. Phys. Anal. Geom. 4 (2001), no. 2, 97-130.

[24] A. Klein, A. Koines and M. Seifert, Generalized eigenfunctions for waves in inhomogeneous media, J. Funct. Anal., to appear.

[25] B. Metzger and P. Stollmann, Heat kernel estimates on weighted graphs, Bull. London Math. Soc. 32 (2000), 477-483.

[26] A. Nagel, E. M. Stein and S. Wainger, Balls and metrics defined by vector fields I: Basic properties, Acta Math. 155 (1985), 103-147.

[27] M. H. Pang, Heat kernels of graphs, J. London Math. Soc. (2) 47 (1993), 50-64.

[28] T. Poerschke and G. Stolz, On eigenfunction expansions and scattering theory, Math. Z. 212 (1993), 337-357.

[29] T. Poerschke, G. Stolz and J. Weidmann, Expansions in Generalized Eigenfunctions of Selfadjoint Operators, Math. Z. 202 (1989), 397-408.

[30] D. W. Robinson, Elliptic Operators and Lie Groups, Oxford University Press, Oxford 1991.

[31] L. Rothschild and E. M. Stein, Hypoelliptic differential Operators and nilpotent Groups, Acta Math. 137 (1976), 247-320.

[32] B. Simon, Schrödinger semigroups, Bull. Amer. Math. Soc. 7 (1982), 447-526.

[33] P. Stollmann, Localization for acoustic waves in random perturbations of periodic media, Israel J. Math. 107 (1998), 125-139.

[34] P. Stollmann, Caught by disorder, bound states in random media, Progr. Math. Phys. 20, Birkhäuser, Boston 2001.

[35] P. Stollmann, Scattering by obstacles of finite capacity, J. Funct. Anal. 121 (1994), 416-425.

[36] P. Stollmann and J. Voigt, A regular potential that is nowhere in $L_{1}$, Lett. Math. Phys. 9 (1985), 227-230

[37] P. Stollmann and J. Voigt, Perturbation of Dirichlet forms by measures, Potent. Anal. 5 (1996), 109-138. 
[38] K. T. Sturm, Analysis on loal Dirichlet spaces I. Recurrence, conservativeness and $L^{p}$ Liouville properties, J. reine angew. Math. 456 (1994), 173-196.

[39] K. T. Sturm, Analysis on local Dirichlet spaces II. Upper Gaussian estimates for the fundamental solution of parabolic equations, Osaka J. Math. 32 (1995), 275-312.

[40] K. T. Sturm, Analysis on local Dirichlet spaces III, J. Math. Pures Appl. 75 (1996), 273-297.

Université Paris-7, Mathématiques, case 7012, 2, place Jussieu, 75251 Paris Cedex 05, France

TU Chemnitz, Fakultät für Mathematik, Reichenhainer Str. 41, 09107 Chemnitz, Germany e-mail: peter.stollmann@mathematik.tu-chemnitz.de

Eingegangen 18. Januar 2002, in revidierter Fassung 24. September 2002 